\title{
How many calories did Santa Claus consume on Christmas Eve?
}

\author{
Gary P. Wormser · Alexander Ladenheim
}

Received: 13 December 2017 / Accepted: 14 December 2017 / Published online: 8 January 2018

(C) Springer-Verlag GmbH Austria, part of Springer Nature 2018

In the twilight hours of Christmas Eve, many families in North America provide cookies and milk for Santa Claus. Avoiding excessive calorie intake is increasingly emphasized as part of a healthy life style [1]. An interesting question is how many calories Santa will have consumed that night on his trip to this region? This topic has been commented on extensively in the last few years (Table 1); however, the methodology used to determine households visited and calories consumed per household varies widely, is often incompletely disclosed, and when it is disclosed, often seems arbitrary. Furthermore, no source we reviewed attempted to address the specific metabolic demands of Santa Claus or the thermic effect of the food he consumes. Here we attempt to construct a more accurate picture of the Calories (herein use of a capital "C" refers to large/ food calories) both ingested and expended by Santa Claus on Christmas Eve 2017. We restrict our data to the United States of America, where survey information is available on pertinent attitudes and behaviors in the population.

In 2013, a Pew survey showed that of United States adults with children, $49 \%$ planned to make preparations for Santa's arrival on Christmas Eve, as did a surprising $22 \%$ of adults who were not parents or guardians [2]. According to the 2016 American Community Survey (United States Census Bureau), there are 118,860,065 households in the USA, with an average size of 2.65 persons. Of these, there were $36,846,620$ households with children $<18$ years of age (31\%; [3]). If $49 \%$ of United States households with children make preparations for Santa's arrival, this yields 18.1 million households. Furthermore, of the households without children, if $22 \%$ prepare for

Dr. G. P. Wormser (西)

New York Medical College, Valhalla, NY 10595, USA

gwormser@nymc.edu
Santa's arrival, this yields an additional 18.0 million households, for a total of 36.1 million households that will leave out cookies and milk (or another suitable snack).

If each home leaves out three cookies, and assuming that the average cookie contains 50-100 Calories, plus an 8-ounce glass of milk ( 135 Calories), Santa can anticipate at least a 275 Calorie or 1150 kiloJoules (kJ) snack waiting for him at each house he visits. Therefore, before the night is out, he will have consumed approximately 9.9 billion Calories ( 41.4 billion $\mathrm{kJ}$ ), just from the visit to the USA. This snack consists mainly of carbohydrates and fat. To account for the thermic effect of food, let us generously assume that $10 \%$ of these Calories are used in the process of digestion, which leaves 8.9 billion Calories (37.2 billion $\mathrm{kJ}$ ). Assuming Santa is the equivalent of a 60 -year-old, $110 \mathrm{~kg}$, active male with a height of $1.8 \mathrm{~m}$, his daily total energy expenditure (TEE) can be calculated as follows [4]:

$$
\begin{gathered}
\text { TEE }=1086-(10.08 \times \text { age })+[1.25 \times(13.7 \times \text { weight } \\
+416 \times \text { height })]=3301 \text { Calories } / \text { day }(13,811 \mathrm{~kJ} / \text { day })
\end{gathered}
$$

Consumption of 8.9 billion Calories would exceed Santa's usual daily requirement by 2.7 million-fold. Even if Santa spread out the consumption over the next 365 days until the following Christmas, the daily Calorie consumption would exceed the standard consumption by over 7300 -fold per day. One might ponder whether these Calories are offset by his travails of the night. Sadly, this is unlikely. For example, let us assume that Santa has to ascend up a $4 \mathrm{~m}$ chimney when he leaves each house. The work required to raise him and his bag of gifts (perhaps $160 \mathrm{~kg}$ in total) up a $4 \mathrm{~m}$ chimney comes to a mere 1.5 Calories $(6.3 \mathrm{~kJ})$, although this translates to more than 54.2 million 
Table 1 Calorie consumption by Santa Claus and parameters published by internet media groups

\begin{tabular}{|c|c|c|c|c|c|}
\hline Source & $\begin{array}{l}\text { Calories con- } \\
\text { sumed in billions } \\
\text { (kiloJoules in } \\
\text { billions) }\end{array}$ & $\begin{array}{l}\text { Households in } \\
\text { millions }\end{array}$ & $\begin{array}{l}\text { Calories/household } \\
\text { (kiloJoules) }\end{array}$ & Methodology (if disclosed) & URL (all accessed December 7, 2017) \\
\hline OC Weekly & $\begin{array}{l}0.262 \\
(1.10)\end{array}$ & 1.14 & $\begin{array}{l}230 \\
(962)\end{array}$ & $\begin{array}{l}\text { USA: } 100 \text { million households, } \\
76 \% \text { Christian, } 15 \% \text { with } \\
\text { children, } 10 \% \text { actually put } \\
\text { out cookies }\end{array}$ & $\begin{array}{l}\text { http://www.ocweekly.com/restaurants/ } \\
\text { how-many-calories-does-santa-claus- } \\
\text { consume-on-christmas-6617835 }\end{array}$ \\
\hline Spoon University & $\begin{array}{l}494 \\
(2067)\end{array}$ & 950 & $\begin{array}{l}520 \\
(2176)\end{array}$ & $\begin{array}{l}\text { Worldwide: } 1.9 \text { billion chil- } \\
\text { dren worldwide, } 2 \text { children/ } \\
\text { household }\end{array}$ & $\begin{array}{l}\text { https://spoonuniversity.com/lifestyle/this- } \\
\text { is-how-many-calories-santa-would- eat- } \\
\text { on-christmas }\end{array}$ \\
\hline USA Today & $\begin{array}{l}7.00 \\
(29.3)\end{array}$ & 20 & $\begin{array}{l}350 \\
(1464)\end{array}$ & USA & $\begin{array}{l}\text { https://www.usatoday.com/videos/news/ } \\
\text { nation/2015/12/23/77835834/ }\end{array}$ \\
\hline Chron & $\begin{array}{l}0.921 \\
(3.85)\end{array}$ & 32.9 & $\begin{array}{l}28 \\
(117)\end{array}$ & $\begin{array}{l}\text { USA: } 61 \text { million children } \\
\text { age } 0-14 \text { years, } 1.86 \text { chil- } \\
\text { dren/household } \\
\text { One "bite" of cookie } \\
\text { (=12 Calories) plus one } \\
\text { "sip" of milk (=16 Calories) }\end{array}$ & $\begin{array}{l}\text { http://www.chron.com/life/health/article/ } \\
\text { The-crazy-number-of-calories-Santa- } \\
\text { Claus-will-eat-6717682.php }\end{array}$ \\
\hline Quora & $\begin{array}{l}105 \\
(439)\end{array}$ & 378 & $\begin{array}{l}278 \\
(1163)\end{array}$ & Worldwide & $\begin{array}{l}\text { https://www.quora.com/How-many- } \\
\text { calories-would-Santa-gain-and-burn- } \\
\text { from-eating-all-those-cookies-and- } \\
\text { delivering-all-those-presents-on- } \\
\text { Christmas }\end{array}$ \\
\hline Eat Out Eat Well & $\begin{array}{l}18.4 \\
(77.0)\end{array}$ & 92 & $\begin{array}{l}200 \\
(837)\end{array}$ & Worldwide & $\begin{array}{l}\text { http://www.eatouteatwell.com/do-all- } \\
\text { those-cookies-create-a-problem-for- } \\
\text { santa/ }\end{array}$ \\
\hline Greatist & $\begin{array}{l}374 \\
(1565)\end{array}$ & 2200 & $\begin{array}{l}170 \\
(711)\end{array}$ & $\begin{array}{l}\text { Worldwide: } 2.2 \text { billion Chris- } \\
\text { tians, each of whom leaves } \\
\text { milk and cookies }\end{array}$ & $\begin{array}{l}\text { https://greatist.com/happiness/santa- } \\
\text { science-facts-christmas- } 122412\end{array}$ \\
\hline Snopes & $\begin{array}{l}20.7 \\
(86.6)\end{array}$ & 91.8 & $\begin{array}{l}225 \\
(941)\end{array}$ & $\begin{array}{l}\text { Worldwide: } 378 \text { million Chris- } \\
\text { tian children, } 3.5 \text { children/ } \\
\text { household }\end{array}$ & $\begin{array}{l}\text { http://www.snopes.com/holidays/ } \\
\text { christmas/santa/physics.asp }\end{array}$ \\
\hline Telegraph & $\begin{array}{l}259 \\
(1084)\end{array}$ & 740 & $\begin{array}{l}350 \\
(1464)\end{array}$ & $\begin{array}{l}\text { Worldwide: } 1.85 \text { billion } \\
\text { children age } 0-14 \text { years, } \\
2.5 \text { children/household; } \\
\text { minced pies and milk }\end{array}$ & $\begin{array}{l}\text { http://www.telegraph.co.uk/finance/ } \\
\text { economics/11307918/Santanomics-the- } \\
\text { calculations-that-make-it-all-possible-for- } \\
\text { Saint-Nick.html }\end{array}$ \\
\hline Daily Mail & $\begin{array}{l}150 \\
(628)\end{array}$ & 640 & $\begin{array}{l}234 \\
(979)\end{array}$ & Worldwide: CEBR $^{\mathrm{a}}$ & $\begin{array}{l}\text { http://www.dailymail.co.uk/sciencetech/ } \\
\text { article-2521973/The-science-Santa-Mr- } \\
\text { Claus-eat-150-BILLION-calories-visit-5- } \\
\text { 556-houses-SECOND-Christmas-Eve.html }\end{array}$ \\
\hline Quick Country & $\begin{array}{l}150 \\
(628)\end{array}$ & 640 & $\begin{array}{l}234 \\
(979)\end{array}$ & Worldwide: $\mathrm{CEBR}^{\mathrm{a}}$ & $\begin{array}{l}\text { http://quickcountry.com/santa-facts-how- } \\
\text { many-calories-does-he-eat/ }\end{array}$ \\
\hline $\begin{array}{l}\text { Mr. Mango } \\
\text { Delivery }\end{array}$ & $\begin{array}{l}156 \\
(653)\end{array}$ & 640 & $\begin{array}{l}244 \\
(1021)\end{array}$ & Worldwide: CEBR $^{\mathrm{a}}$ & $\begin{array}{l}\text { http://mrmangodelivery.co.uk/blog/how- } \\
\text { many-calories-does-santa-eat-on- } \\
\text { christmas-eve/ }\end{array}$ \\
\hline Z1035 & $\begin{array}{l}150 \\
(628)\end{array}$ & 640 & $\begin{array}{l}234 \\
(979)\end{array}$ & Worldwide: CEBR $^{\mathrm{a}}$ & $\begin{array}{l}\text { http://z1035.com/how-much-santa-will- } \\
\text { eat-drink-on-christmas-eve/ }\end{array}$ \\
\hline
\end{tabular}

Calories (227 million $\mathrm{kJ}$ ) expended across all of the households visited in the USA.

Although it is likely that some of the assumptions we made were incorrect, precluding a precise determination of the number of Calories that were consumed by Santa Claus on this past Christmas Eve, it is, nevertheless, quite apparent that the number would be enormous by any usual standard! But Santa does not gain weight and returns year after year seemingly at about the same weight. That Santa might have an extraordinarily high rate of metabolism, or perhaps had bariatric surgery that unexpectedly resulted in a profound degree of malabsorption, does not com- port with his stable weight and apparent good health. Instead, we can only conclude that it's a Christmas miracle!

Conflict of interest G.P. Wormser reports receiving research grants from Immunetics, Inc., Institute for Systems Biology, Rarecyte, Inc., and Quidel Corporation. He owns equity in Abbott/AbbVie; has been an expert witness in malpractice cases involving Lyme disease; and is an unpaid board member of the American Lyme Disease Foundation. A. Ladenheim declares that he has no competing interests. 


\section{References}

1. Centers for Disease Control and Prevention. Healthy eating for a healthy weight. 2016. https://www.cdc.gov/ healthyweight/effects/index.html. Accessed 7 Dec 2017.

2. Pew Research Center. Celebrating christmas and the holidays, then and now. 2013. http://www.pewforum.org/ 2013/12/18/celebrating-christmas-and-the-holidaysthen-and-now/ (Created 18 Dec 2013). Accessed 7 Dec2017.
3. US Census Bureau. American Community Survey (ACS) 1-year estimates: "table S1101-households and families". 2016.

4. Institute of Medicine of the National Academies. Dietary reference intakes for energy, carbohydrate, fiber, fat, fatty acids, cholesterol, protein, and amino acids. Washington, DC: The National Academies Press; 2005. https://doi.org/ $10.17226 / 10490$. 